\title{
Umfrage zur Weiterbildung in der Interventionellen Radiologie
}

Das Forum Junge Radiologie hat in Zusammenarbeit mit der DeGIR eine Umfrage für Weiterbildungsassistentinnen und -assistenten initiiert, die Genaueres über die Weiterbildung in der Interventionellen Radiologie in Erfahrung bringen möchte.

Das Forum Junge Radiologie hat in den vergangenen Jahren bereits zweimal eine allgemeine Weiterbildungsumfrage durchgeführt, die auf ein sehr positives Echo gestoßen ist. Es konnte gezeigt werden, dass die Radiologie bereits einen sehr guten Weiterbildungsstandard hat, aber auch Verbesserungspotentiale bestehen.

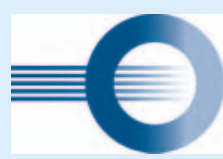

\section{Forum Junge Radiologie}

in der Deutschen Röntgengesellschaft e.V.

Die aktuelle Umfrage konzentriert sich nun auf den Bereich „Interventionellen Radiologie“ und wurde gemeinsam mit der DeGIR erarbeitet. Dabei soll neben allgemeinen Fragen zu den bestehenden Rahmenbedingungen, speziell der interventionellen Weiterbildung und möglichen Verbesserungspotentialen, auch ein besonderer Fokus auf die Situation weiblicher Radiologinnen gelegt werden.
Sind Sie Weiterbildungsassistent oder -assistentin? Dann gelangen Sie hier zur Umfrage: https://www.surveymonkey. de/r/PQNHF9W. Geöffnet ist sie bis zum 30. November 2020.

Die Ergebnisse sollen auf dem Deutschen Röntgenkongress 2021 - aber auch weiteren Kongressen - und natürlich auf https:// www.forum-junge-radiologie.de/ vorgestellt werden. 